\title{
BASECOL2012: A collisional database repository and web service within the Virtual Atomic and Molecular Data Centre (VAMDC)
}

\author{
M.-L. Dubernet ${ }^{1,2}$, M. H. Alexander ${ }^{3}$, Y. A. Ba ${ }^{1}$, N. Balakrishnan ${ }^{4}$, C. Balança ${ }^{5}$, C. Ceccarelli ${ }^{6}$, J. Cernicharo ${ }^{7}$,

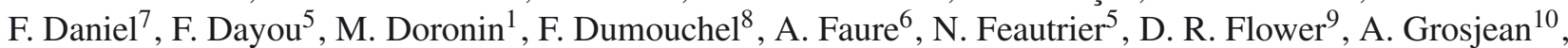 \\ P. Halvick ${ }^{11}$, J. Kłos ${ }^{2}$, F. Lique ${ }^{8}$, G. C. McBane ${ }^{12}$, S. Marinakis ${ }^{1}$, N. Moreau ${ }^{5}$, R. Moszynskii ${ }^{13}$, D. A. Neufeld ${ }^{14}$, \\ E. Roueff ${ }^{15}$, P. Schilke ${ }^{16}$, A. Spielfiedel ${ }^{5}$, P. C. Stancil ${ }^{17}$, T. Stoecklin ${ }^{11}$, J. Tennyson ${ }^{18}$, B. Yang ${ }^{17}$, \\ A.-M. Vasserot ${ }^{1}$, and L. Wiesenfeld ${ }^{6}$ \\ ${ }^{1}$ LPMAA, CNRS UMR 7092, Université Pierre et Marie Curie, Case 76, 4 Place Jussieu, France \\ e-mail: marie-lise.dubernet@obspm.fr \\ 2 Observatoire de Paris-Meudon, 5 Place Janssen, 92195 Meudon Cedex, France \\ 3 Department of Chemistry and Biochemistry, University of Maryland, College Park, MD 20742, USA \\ ${ }^{4}$ University of Nevada-Las Vegas, Las Vegas, NV 89154, USA \\ 5 LERMA, CNRS UMR 8112, Observatoire de Paris, 5 Place Janssen, 92195 Meudon, France \\ ${ }^{6}$ UJF-Grenoble 1/CNRS-INSU, Institut de Planétologie et d'Astrophysique de Grenoble (IPAG), UMR 5274, 38041 Grenoble, \\ France \\ 7 CAB. INTA-CSIC, Crta. Torrejón km 4, 28850 Torrejón de Ardoz, Madrid, Spain \\ ${ }^{8}$ Laboratoire Ondes et Milieux Complexes, CNRS UMR 6294, Université du Havre, 25 rue Philippe Lebon, BP 540, \\ 76058 Le Havre, France \\ 9 Physics Department, The University of Durham, Durham, DH1 3LE, UK \\ 10 Institut UTINAM, UMR CNRS 6213, 41 bis avenue de l'Observatoire, BP 1615, 25010 Besançon Cedex, France \\ 11 Institute des Sciences Moléculaires, CNRS UMR 5255, Université Bordeaux I, 351 Cours de la Libération, 33405 Talence, France \\ 12 Department of Chemistry, Grand Valley State University, Allendale, Michigan 49401, USA \\ 13 Quantum Chemistry Laboratory, Department of Chemistry, University of Warsaw, Pasteura 1, 02-093 Warsaw, Poland \\ 14 Department of Physics and Astronomy, The Johns Hopkins University, 3400 North Charles St., Baltimore, MD 21218, USA \\ 15 LUTH, Observatoire de Paris, 5 Place Janssen, 92195 Meudon, France \\ 16 I. Physikalisches Institut der Universität zu Köln, Zülpicher Str. 77, 50937 Köln, Germany \\ 17 Depart. of Physics and Astronomy and Center for Simulational Physics, The University of Georgia, Athens, Georgia 30602-2451, \\ USA \\ 18 Department of Physics and Astronomy, University College London, London WC1E 6BT, UK
}

Received 25 October 2012 / Accepted 4 March 2013

\begin{abstract}
The BASECOL2012 database is a repository of collisional data and a web service within the Virtual Atomic and Molecular Data Centre (VAMDC, http: //www . vamdc . eu). It contains rate coefficients for the collisional excitation of rotational, ro-vibrational, vibrational, fine, and hyperfine levels of molecules by atoms, molecules, and electrons, as well as fine-structure excitation of some atoms that are relevant to interstellar and circumstellar astrophysical applications. Submissions of new published collisional rate coefficients sets are welcome, and they will be critically evaluated before inclusion in the database. In addition, BASECOL2012 provides spectroscopic data queried dynamically from various spectroscopic databases using the VAMDC technology. These spectroscopic data are conveniently matched to the in-house collisional excitation rate coefficients using the SPECTCOL sofware package (http:// vamdc.eu/software), and the combined sets of data can be downloaded from the BASECOL2012 website. As a partner of the VAMDC, BASECOL2012 is accessible from the general VAMDC portal (http://portal.vamdc. eu) and from user tools such as SPECTCOL.
\end{abstract}

Key words. molecular data - virtual observatory tools - astronomical databases: miscellaneous - ISM: molecules - scattering molecular processes

\section{Introduction}

Since the discovery of the first molecule in the interstellar medium (ISM) some fifty years ago, the number of detected molecules has steadily increased with time to more than 200 molecules nowadays ${ }^{1}$. The study of ISM molecules has various motives. First, it has been shown that a relatively rich

\footnotetext{
E.g. see the list at www. astrochymist.org/ and www . astro.uni-koeln. de/cdms/molecules
}

chemistry can occur in the ISM, where the conditions are rather harsh in terms of temperature and density when compared to those on Earth. This chemistry mainly forms small diatomic molecules but also complex polyatomic molecules, the largest of them organic, which leads to the unanswered question of what might have been the role of ISM molecules in the terrestrial life appearance. Second, molecules are extremely important for probing the physical conditions of the gas, thanks to the fact that molecular lines are formed under particular temperatures and 
densities that depend on the precise molecule structure and observed line. As a consequence, multifrequency observations can be used to reconstruct the physical structure of the studied object. However, in practice this is only possible if the so-called "collisional rate coefficients" are known. In the vast majority of the conditions present in the ISM, these coefficients are indispensable i) to convert an observed signal into a species column density and, therefore, abundance; and ii) to constrain the density and temperature of the emitting or absorbing gas. To this end, the molecular dynamics/quantum chemistry communities have been (and still are) extremely active in providing accurate collisional rate coefficients values (Dubernet et al. 2011).

With the advent of a new generation of heterodyne instruments possessing large instantaneous bandwidths, from the ground-based single-dish 30m IRAM to the Atacama Large Millimeter/submillimeter Array (ALMA) interferometer, and to the Herschel Space Observatory, the simultaneous observations of several molecular transitions is routine nowadays. In fact, unbiased spectral surveys are becoming a privileged tool for studying the chemical and physical structure of astrophysical sources (Bergin et al. 2010; Ceccarelli et al. 2010; Caux et al. 2011; Patel et al. 2011; Tercero et al. 2011; Ginard et al. 2012; Zernickel et al. 2012). Finally, the ever-increasing sensitivity of receivers will also lead to an increasing number of molecular lines to interpret. The huge investment in these new facilities, such as the Herschel-Heterodyne Instrument for the Far-Infrared (HIFI) (de Graauw et al. 2010) or the ALMA interferometer ${ }^{2}$, therefore, makes the knowledge and availability of the collisional coefficients essential. In fact, in this context, having easy access to database of collisional coefficients has become more than an urgent need: it is a must.

The BASECOL database was first established at Besançon Observatory in 2002 in order to provide a compilation of references related to inelastic rate coefficients for atoms and molecules detected in ISM. The next version, BASECOL2005 (Dubernet et al. 2006) hosted at Paris Observatory (basecol.obspm.fr), was extended to include rate coefficients for some molecular species colliding with atoms and molecules. This version was accessible via its conventional web interface and via an automatic procedure for retrieving data (Moreau \& Dubernet 2006a,b). The Leiden LAMDA database was established at the same time (Schöier et al. 2005).

BASECOL works on the concept of a collisional system comprising an atomic or molecular target species that contains internal structure and a projectile, which in general is assumed to be unstructured; however, this is not always the case, as seen in the discussion of collisions with $\mathrm{H}_{2}$ in Sect. 2.2.1. The temperature used for the rate coefficients is the relative translation of the two colliders, while the target states are generally treated as being fully resolved.

Since the previous editions of BASECOL, fitted functions for many available rate coefficients have been added, collisional systems involving electrons have been included, and there have been continuing updates of the database with the latest published results for collisional systems of interest. However, the greatest conceptual change is the implementation of BASECOL2012 within the Virtual Atomic and Molecular Data Center $^{3}$ (Dubernet et al. 2010; Endres et al. 2011; Rixon et al. 2011).

The primary goal of the BASECOL database is to provide a standard repository of collisional rate coefficients for use by

\footnotetext{
2 http://www.almaobservatory.org/

3 www. vamdc.eu
}

the scientific community. To ensure data quality, inclusion in BASECOL requires the team to critically evaluate any input data by reading the relevant papers and, when necessary, communicating with the original authors. Currently, BASECOL provides collisional data sets as originally published only and archives of earlier datasets for each given collisional system, thus providing long-term preservation of the data. For guidance to users, BASECOL indicates some sets as "recommended" based on an evaluation by the BASECOL team.

It should be noted that at present BASECOL does not contain experimental rate coefficients because those data are rarely obtained directly. Indirect measurements, such as linebroadening coefficients, are not relevant to BASECOL since it is hard to extract accurate collisional excitation rate coefficients from such measurements, see Drouin et al. (2010), Krupnov (2010), Wiesenfeld \& Faure (2010), and finally Drouin \& Wiesenfeld (2012) as an illustrative example. Nevertheless, indirect measurements of line broadening, line-shifting coefficients, or bound states of Van der Waals systems can give an assessment of the accuracy of some regions of the potential energy surfaces used in the heavy particle scattering calculations.

The database has a "Data" section that includes: a) Collision browser or search engine sections that give access to the collisional systems linking to collisional rate coefficients combined with experimental spectroscopic information and a brief description of the theoretical methodology used. b) Information on the energy levels used in the molecular dynamics calculations: this section is aimed at theoreticians who want to reproduce the rate coefficient calculations.

A "Bibliographic" section provides access to the bibliographic databases, and the "Tools" section provides tools that might be of interest to the astrophysical community, in particular the SPECTCOL tool (Dubernet et al. 2012; Dubernet \& Nenadovic 2011) as well as some code packages to handle BASECOL data sets. A "Submit new data" section provides instructions about how to submit new collisional data sets. A "Publication" section contains publications associated to BASECOL, i.e. reviewed papers, proceedings, etc. The "Field news" section is meant to display information on current developments and future needs, the "Support" section provides information about how to register to the basecol-user list.

\section{Overview of the BASECOL database}

\subsection{A bibliographic database on molecular energy transfer}

Currently there are a total of 829 references in the bibliographic database, which is updated regularly. These references are mainly linked to the collisional data sets entered in BASECOL. They range from purely theoretical papers on dynamical or quantum chemistry methods to papers describing potential energy surfaces, collisional cross sections, and rate coefficients. In addition to a standard bibliographic search engine, a search via in-house keywords is provided since all papers have been indexed with keywords relevant for the inelastic collisions contained in BASECOL. Whenever possible, links to the web pages that have the original publications or to the SAO/NASA Astrophysics Data System (ADS) are also provided. The resulting citations can be extracted in BibTeX format.

\subsection{Collisional systems}

The BASECOL2012 database gathers and keeps information on collisional systems. This means that for a given collisional system, the users have access to sets calculated by different authors 
or by the same authors at different times. BASECOL2012 has the policy of not scaling the collisional rate coefficients of moleculeHe systems and pretending that such scaled data can replace the corresponding molecule- $\mathrm{H}_{2}$ data. It also does not scale a given neutral-neutral system to mimic another isoelectronic system. BASECOL2012 directly gets its spectroscopic data dynamically from the CDMS, JPL, HITRAN databases and keeps their own version. BASECOL2012 keeps a version of its own internal data sets. BASECOL collisional data allows for the possibility of state-to-state rate coefficients including transitions in a structured collider. Such a possibility is rich from the physical point of view, but might both complicate the lives of users and be considered as a feature of BASECOL. Therefore Sect. 2.2.1 provides the necessary explanations and recommendations. All these aspects define the difference between the BASECOL2012 and the LAMDA (Schöier et al. 2005) databases.

Another feature of BASECOL arose because users encountered difficulties choosing between different collisional sets for a given system, therefore a "recommended" mention is added to BASECOL2012 (see Sect. A.7). A long-lasting demand on BASECOL has been to provide combined collisional and spectroscopic data in the format used by the radiative transfer code RADEX (van der Tak et al. 2007), which is also used by the LAMDA database (Schöier et al. 2005). Therefore, BASECOL2012 has added this new feature in the "Radiation/Collision" section of the collisional sets. Table A.1 summarizes the information available for each collisional set.

\subsubsection{Rate coefficients}

The collisional rate coefficients provided by BASECOL are state-to-state rate coefficients, effective rate coefficients, and thermalized rate coefficients, each of which is defined below. In most cases state-to-state (de-)excitation rate coefficients are obtained at a given temperature from Boltzmann thermal averages of the calculated state-to-state inelastic cross-sections obtained on a grid of kinetic energies $E$ :

$$
\begin{array}{r}
R\left(\alpha \rightarrow \alpha^{\prime} ; \beta \rightarrow \beta^{\prime}\right)(T)= \\
\left(\frac{8}{\pi \mu}\right)^{1 / 2} \frac{1}{\left(k_{\mathrm{B}} T\right)^{3 / 2}} \int_{0}^{\infty} \sigma_{\alpha \rightarrow \alpha^{\prime} ; \beta \rightarrow \beta^{\prime}}(E) E e^{-E / k_{\mathrm{B}} T} \mathrm{~d} E,
\end{array}
$$

where $k_{\mathrm{B}}$ the Boltzmann constant, $\mu$ the reduced mass of the colliding system, and $(\alpha, \beta),\left(\alpha^{\prime}, \beta^{\prime}\right)$ represent the initial and final levels of the target $(\alpha)$ and collider $(\beta))$ species. Therefore a typical BASECOL rate coefficient table is composed of the following items: Cols. 1 and 2 contain the initial $\alpha$ and final $\alpha^{\prime}$ levels of the target, Cols. 3 and 4 contain the initial $\beta$ and final $\beta^{\prime}$ levels of the collider, the subsequent columns give the state-to-state rate coefficients (Eq. (1)) at different temperatures.

These state-to-state collisional rate coefficients follow the principle of detailed balance, and reverse rate coefficients $R\left(\alpha^{\prime} \rightarrow \alpha ; \beta^{\prime} \rightarrow \beta\right)(T)$ can be obtained from forward rate coefficients by the usual formula:

$$
\begin{array}{r}
g_{\alpha^{\prime}} g_{\beta^{\prime}} e^{-\frac{E_{\text {int }}^{\prime}\left(\alpha^{\prime}\right)}{k_{\mathrm{B}} T}} e^{-\frac{E_{\text {int }}^{\prime}\left(\beta^{\prime}\right)}{k_{\mathrm{B}} T}} R\left(\alpha^{\prime} \rightarrow \alpha ; \beta^{\prime} \rightarrow \beta\right)= \\
g_{\alpha} g_{\beta} e^{-\frac{E_{\mathrm{int}}(\alpha)}{k_{\mathrm{B}} T}} e^{-\frac{E_{\mathrm{in}}(\beta)}{k_{\mathrm{B}} T}} R\left(\alpha \rightarrow \alpha^{\prime} ; \beta \rightarrow \beta^{\prime}\right),
\end{array}
$$

where $g_{\alpha}$ and $g_{\beta}$ are the statistical weights related to the rovibrational levels of the target and collider, respectively, and the different $E_{\text {int }}$ are the ro-vibrational energies of the species.

When the collider is an electron or an atom whose internal energy does not change during the collision, the state-to-state (de)-excitation rate coefficient is Eq. (1) with $\beta=\beta^{\prime}=1$. For ISM studies, the usual atomic colliders are $\mathrm{He}, \mathrm{H}, \mathrm{H}^{+}$, whose internal states do not change during the collision for kinetic energies relevant to ISM studies.

When the collider is a molecule, such as $\mathrm{H}_{2}$, no such simplification occurs rigorously because the first transition in the rotational levels of para- $\mathrm{H}_{2}$ and ortho- $\mathrm{H}_{2}$ occurs around $354 \mathrm{~cm}^{-1}$ and $587 \mathrm{~cm}^{-1}$, respectively. Nevertheless, except for the latest $\mathrm{H}_{2} \mathrm{O}-\mathrm{H}_{2}$ calculations of Daniel et al. $(2011,2010)$ and Dubernet et al. (2009), where excitation of $j\left(\mathrm{H}_{2}\right)=2,3,4$ are considered, and for the $\mathrm{SiS}-\mathrm{H}_{2}$ system (Kłos \& Lique 2008), HNC$\mathrm{H}_{2}$ (Dumouchel et al. 2011), and $\mathrm{CN}^{-}-\mathrm{H}_{2}$ (Kłos \& Lique 2011) where rate coefficients with $j\left(\mathrm{H}_{2}\right)=2$ were provided, nearly all published calculations do not allow excitation of $\mathrm{H}_{2}$, thereby fixing $\mathrm{H}_{2}$ in its lowest para $(j=0)$ and ortho $(j=1)$ states. Within this approximation, which is usually but not always justified in the ISM, the state-to-state (de)-excitation rate coefficient is Eq. (1) with $\beta=\beta^{\prime}=1$ as for atoms.

State-to-state rate coefficients that include excitation of the colliding $\mathrm{H}_{2}$ (Daniel et al. 2011, 2010; Dubernet et al. 2009) are not directly usable in the current astrophysics radiative transfer codes since these codes do not explicitly consider excitation of the collider. In this case it is necessary to use the so-called effective rate coefficients $\hat{R}_{\beta}\left(\alpha \rightarrow \alpha^{\prime}\right)$, which are given by the sum of the state-to-state rate coefficients (Eq. (1)) over final collider states, $\beta^{\prime}$ for a given initial $\beta$ :

$\hat{R}_{\beta}\left(\alpha \rightarrow \alpha^{\prime}\right)(T)=\sum_{\beta^{\prime}} R\left(\alpha \rightarrow \alpha^{\prime} ; \beta \rightarrow \beta^{\prime}\right)(T)$.

These effective rate coefficients do not follow the principle of detailed balance, so both excitation and de-excitation rates should be calculated explicitly. In BASECOL the effective rate coefficients are clearly identified as "Effective" and the table's entry for the collider initial level indicates the $\beta$ level of Eq. (3), while the collider final level is meaningless and is currently set equal to the initial level for convenience.

Finally, de-excitation rate coefficients for a thermalized collider can be obtained by averaging over the initial ro-vibrational levels of the collider:

$\bar{R}\left(\alpha \rightarrow \alpha^{\prime}\right)(T)=\sum_{\beta} \rho(\beta) \hat{R}_{\beta}\left(\alpha \rightarrow \alpha^{\prime}\right)(T)$

with $\rho(\beta)=g_{\beta} e^{-\frac{E_{\text {int }}(\beta)}{k_{\mathrm{B}} T}} / Z$, where $Z(T)$ is the partition function obtained as sum over the $\beta$ states. Such rate coefficients follow the principle of detailed balance.

For colliders having ortho/para species such as $\mathrm{H}_{2}$, the user will consider the two species as independent in most cases. It should be noted that quasi-classical calculations (QCT) calculations, such as those of Faure et al. (2007b), directly calculate the rotational de-excitation rate coefficients of $\mathrm{H} 2 \mathrm{O}$ by thermalized ortho/para $\mathrm{H}_{2}$ considered as separate species. In BASECOL the thermalized rate coefficients are clearly identified as "Thermalized" and the entries for the collider's initial and final levels are meaningless. They are always denoted the $\beta=\beta^{\prime}=1$ level for convenience.

It has been found that for all collisional systems being studied the state-to-state or effective rate coefficients with $j\left(\mathrm{H}_{2}\right)=$ 1 are always higher than those with $j\left(\mathrm{H}_{2}\right)=0$, with the exception of excitation of the negative ion $\mathrm{CN}^{-}$(Kłos \& Lique 2011) where there are no significant differences between the collisions with the two species of $\mathrm{H}_{2}$. Furthermore, it was shown for $\mathrm{H}_{2} \mathrm{O}-\mathrm{H}_{2}$ (Daniel et al. 2011, 2010; Dubernet et al. 2009) that 
the effective rate coefficients for $j\left(\mathrm{H}_{2}\right)=2,3,4$ are close to the $j\left(\mathrm{H}_{2}\right)=1$ effective rate coefficients. It is probably safe to assume that this is a quasi-general feature. Therefore in the absence of data for $j\left(\mathrm{H}_{2}\right)$ greater than 1 , thermalized quantum rate coefficients can be estimated using the effective rate coefficients with $j\left(\mathrm{H}_{2}\right)=0,1$ replacing all others with $j\left(\mathrm{H}_{2}\right)=1$ effective rate coefficients.

\subsubsection{Methodologies for collisions with heavy particles}

The current heavy-particle BASECOL data is mostly based on collisional calculations performed within the BornOppenheimer approximation, which leads to a three-step procedure: calculation of the so-called potential energy surfaces (PES) via ab initio quantum chemistry or empirical methods, representation of the PES by some functional form useful for the dynamical calculations, and finally dynamical calculations. For some specific systems like open-shell radical $(\mathrm{OH}, \mathrm{NO}, \mathrm{CH})$ in collision with $\mathrm{He}$ and $\mathrm{H}_{2}$ or open-shell atoms $(\mathrm{F}, \mathrm{Cl})$ in collision with $\mathrm{He}$ and $\mathrm{H}_{2}$, the collision may induce non adiabatic transitions between different spin-orbit states (Baer 1975; Alexander 1985). In these case, non-Born-Oppenheimer effects have to be taken into account in the dynamical calculations but the general methodology stays the same as for collisions described within the Born-Oppenheimer approximation.

The field of heavy particle molecular collisions underwent intensive development in the 1960s and 1970s. Reviews by Light (1979), Kouri (1979), and Secrest (1979) provide an introduction to quantum close coupling (CC) calculations (Arthurs \& Dalgarno 1960) and quantum decoupling approximate methods such as coupled states methods (CS) (McGuire \& Kouri 1974; Pack 1974), decoupled $l$-dominant (DLD) approximation (DePristo \& Alexander 1976) and effective Hamiltonian methods (Rabitz 1976). The infinite order sudden (IOS) approximation (Tsien \& Pack 1970a,b; Secrest 1975), which combines centrifugal decoupling and the sudden approximation, is often used when other quantum methods are too computationally demanding. These methods have been implemented in the publicly available numerical code MOLSCAT (Hutson \& Green 1994; McBane 2004), which is mainly applicable to collisions between closed-shell species ranging from atom-diatom to diatom-asymmetric molecule scattering, and in the codes HIBRIDON (Dagdigian \& Alexander 2011) ${ }^{4}$ and MOLCOL (Flower et al. 2000), which are designed to treat both open and closed shell systems. It is generally accepted that $\mathrm{CC}$ methods are the most accurate ones available, followed by CS, IOS, DLD, and effective Hamiltonian methods. All these methods can calculate state-to-state collisional cross-sections as a function of the relative kinetic energy of the colliding species. Alternatively approaches involving classical or semi-classical approximations are available; the most common approach is the quasi-classical trajectory (QCT) method (Pattengill 1979 and references herein), which solves the classical Hamilton equations using a random (Monte Carlo) sampling of initial conditions combined with a pseudo-quantization of internal energies.

\footnotetext{
4 HIBRIDON is a package of programs for the time-independent quantum treatment of inelastic collisions and photodissociation written by M. H. Alexander, D. E. Manolopoulos, H.-J. Werner, B. Follmeg, Q. Ma, and P. J. Dagdigian, with contributions by P. F. Vohralik, D. Lemoine, G. Corey, R. Gordon, B. Johnson, T. Orlikowski, A. Berning, A. Degli-Esposti, C. Rist, B. Pouilly, G. van der Sanden, M. Yang, F. de Weerd, S. Gregurick, J. Klos and F. Lique. More information and/or a copy of the code can be obtained from the website http://www2 . chem. umd. edu/groups/alexander/hibridon/hib43.
}

If the initial and final quantum states of the species are selected, state-to-state collisional cross sections can be computed as for the above quantum methods. The field of molecular collisional for the ISM owns a tribute to the work of Sheldon Green whose entire work can be found in the BASECOL database. Some other relevant references for molecular collision in the ISM can be found in Flower (2007).

The dynamical methods used nowadays have not evolved significantly since the 1970 s, as reflected by the methodology information given in BASECOL: see Table A.1 and information sections of BASECOL. Some studies have used a combination of methods, for example, the coupled states method for rotational excitation, and the IOS method for the hyperfine excitation, denoted $\mathrm{rCS} / \mathrm{hIOS}$. In other cases, CC is used for low collision energies, and the CS approximation is employed at higher collision energies, denoted CC/CS. If the quantum-mechanical method employed cannot be categorized using the schemes mentioned above, the catch-all notation QM is used.

The increase in computational power has allowed both the treatment of more complex systems and an increase in the accuracy of the dynamical methods used in the calculations. Quantum chemistry calculations have evolved tremendously thanks both to new theoretical developments and to the availability of increasing computer power, making it possible to calculate highly accurate PES. Recently the most popular methods for the closed shell systems included in BASECOL are at the level of the coupled cluster method with single, double, and noniterative triple excitations $(\operatorname{CCSD}(\mathrm{T}))$ calculations with large basis sets as implemented in many quantum chemistry packages, see for instance MOLPRO (Werner et al. 2012) or use symmetryadapted perturbation theory (SAPT) methodologies (Jeziorski et al. 1994; Bukowski et al. 2008). The latter approach has an advantage since the long-range behaviour is fixed at the same level of the theory and on the same basis set (Heijmen et al. 1996). The increase in computer power has also allowed an increase in the number of nuclear geometries spanning the potential energy surface's parameter space, giving an improved description of strongly anisotropic systems and of the long-range part of potential energy surfaces. Nevertheless, for highly anisotropic systems or for systems with many parameters, real difficulties remain with the representation of the highly accurate, calculated ab initio points by functional forms suitable for the dynamical calculations. Poor fits can lead to a loss of accuracy in the functional representation, although there has been some recent progress in this area (Braams \& Bowman 2009).

The accuracy of the ab initio potentials can mostly be checked easily by comparing the measured and computed highresolution spectra, state-to-state cross sections, or rate constants. Some information can also be obtained by comparing the experimental and theoretical differential cross sections, but here the experimental data are usually not state-resolved, and thus are less sensitive to the fine details of the potential. It has been already demonstrated in the 1990s that the ab initio potentials for simple atom-molecule systems like He-CO (Heijmen et al. 1997b) or $\mathrm{Ar}^{-\mathrm{CH}_{4}}$ (Heijmen et al. 1999b; Miller et al. 1999) reproduce the experimental high-resolution spectra very well. For some other systems, such Ne-CO (Moszynski et al. 1997) or $\mathrm{Ne}-\mathrm{C}_{2} \mathrm{H}_{2}$ (Bemish et al. 1998), small scalings were necessary to quantitatively reproduce the experimental data. At the same time, it was shown that the ab initio potentials reproduce the (scarce) data for the state-to-state cross sections and rate constants; see, for instance, Heijmen et al. (1997a) and Heijmen et al. (1999a), respectively. Over the past decade, the complexity of the systems that can be treated by ab initio methods has 
increased dramatically; see for instance, Bukowski et al. (2007) and Jankowski et al. (2012), who show the accuracy that can be reached for systems like the water dimer or $\mathrm{CO}-\mathrm{H}_{2}$.

\subsubsection{Methodologies for collisions with electrons}

The ro-vibrational excitation of molecules by collisions with electrons is a very active field, both experimentally and theoretically. Several theoretical methods and approximations are available, and the interested reader is referred to review papers on electron impact rotational (Itikawa \& Mason 2005) and vibrational (Itikawa 2004) excitation. The theoretical procedure differs somewhat from those used for heavy particle collisions since special methods are required to treat the continuum electron. As far as nuclear motion is concerned, the adiabatic-nuclei (AN) approximation, which is similar in spirit to the IOS approximation, has been widely employed to compute rotational and vibrational cross sections (Gianturco \& Jain 1986), but is only valid away from resonances where the scattering electron is temporarily trapped to form a quasibound state. The AN approximation has been combined with the R-matrix method to compute inelastic rate coefficients for a variety of astrophysically important molecules, see work by Tennyson and co-workers (Faure \& Tennyson 2001; Varambhia et al. 2009) and references therein.

The R-matrix method addresses the electron collision problem by splitting space into two regions: an the inner region where the scattering electron is close to the target, and an outer or longrange region. The wave function in the inner region is expanded in terms of bound and continuum orbitals in a fashion similar to quantum chemistry, whereas a set of close-coupling equations are solved in the outer region. The method has recently been reviewed by Tennyson (2010). For polar species, Born (for neutrals) and Coulomb-Born (for molecular ions) corrections, denoted in Table A.1 as R+Born, are required to account for high partial wave contributions not included in the R-matrix calculations. It is necessary to correct the unphysical behaviour of cross sections at rotational thresholds that are assumed to be degenerate in the AN approximation. For more details, the reader is referred to Varambhia et al. (2009) and references therein. For example, for electron collisions with water, very good agreement is observed with various experimental data once allowance is made for difficulties with measuring forward scattering (Faure et al. 2004b; Zhang et al. 2009). In the case of molecular ions, $\mathrm{R}+$ Born calculations have been shown to be accurate, down to very low energies, by comparison with ro-vibrational quantum defect calculations (Faure et al. 2006) and storage ring measurements (Shafir et al. 2009). Alternatively rate coefficients for rovibrational excitation of molecular cations by electronic impact can be obtained through the multichannel quantum defect theory (MQDT) as recently done for $\mathrm{H}_{3}^{+}$(Kokoouline et al. 2010).

\subsubsection{Fitted and analytic functions of the rate coefficients}

BASECOL contains the original published fits to the collisional rate coefficients, when those are available, or provides in-house fits to the published data. The fitting functions as a function of temperature $T$ are provided, and tabulated fitted parameters can be downloaded. In addition a graphical interface allows the user to plot a comparison between the fitted functions and the original data.

The in-house fitting procedure is a part of the quality assessment of the collisional data. Indeed the procedure ensures that detailed balance is respected between interpolation points. As a general rule, it is important to emphasize that these fits have no physical meaning, and are only valid in the temperature range where the data have been calculated; in particular, they should not be used to perform extrapolations. The in-house fitting is provided on request except if authors oppose the idea of providing fits of their data.

\subsubsection{Coupling to experimental spectroscopic data as a service to the ISM community}

Studies of astrophysical non-LTE media require a combination of collisional and spectroscopic data. Spectroscopic data is provided by databases such as the Cologne Database for Molecular Spectroscopy database $\left(\mathrm{CDMS}^{5}\right)$ (Müller et al. 2001, 2005), the Jet Propulsion Laboratory catalogue (JPL ${ }^{6}$ ) (Pickett et al. 1998), and the HIgh-resolution TRANsmission molecular absorption database (HITRAN ${ }^{7}$ ) (Rothman et al. 2009). As a service to the astrophysical community, the SPECTCOL tool (Dubernet et al. 2012; Dubernet \& Nenadovic 2011) can be used to associate BASECOL collisional data with spectrocopic data taken from the databases listed above for each BASECOL target. Matched spectroscopic data are available for each collisional system in the sections "Labelling" and 'Einstein". If no spectroscopic data are included, either those will be introduced in the future or no spectroscopic data was found. In the latter case we will try to propose some alternatives based on published spectroscopic data. The last column of Table A.1 indicates which collisional sets are matched with spectroscopic data. Versions of the spectroscopic databases are monitored each week, and updated sets of matched spectroscopic data are generated whenever the spectroscopic data are updated. The different versions of matched queried spectroscopic data are kept for reference and can be retrieved. The collisional data and the matched spectroscopic data can be downloaded either separately in BASECOL format or as a single file with the RADEX format (van der Tak et al. 2007) which is often employed by ISM modellers. We note, however, that RADEX format does not allow for any change of state of the collider and only provides de-excitation rate coefficients. This prevents the user from checking that the calculated rate coefficients satisfy a detailed balance. Therefore our RADEX outputs only keep the initial $\alpha$ and final $\alpha^{\prime}$ levels $\left(\alpha>\alpha^{\prime}\right)$ of the target molecule from $R\left(\alpha \rightarrow \alpha^{\prime} ; \beta \rightarrow \beta^{\prime}\right)(T)$ (Eq. (1)), and provide information on the state of the collider. For calculations of state-to-state rate coefficients where the collider is kept in a given state, the state is $\beta=\beta^{\prime}=1$. For effective rate coefficients $\hat{R}_{\beta}\left(\alpha \rightarrow \alpha^{\prime}\right)(T)$ (Eq. (3)) the state of the collider indicates that $\beta$ can be different from 1. For calculations of state-to-state rate coefficients where the internal state of the collider changes, our RADEX outputs keep the state-to-state rate coefficients corresponding to the collider's elastic process alone, i.e. only those transitions corresponding to $\beta=\beta^{\prime}$, and we indicate the value of $\beta$.

\subsection{Assessment and recommendation}

Column 10 of Table A.1 indicates whether the data are recommended or not, denoted "yes" and "no", respectively. These recommendations are reviewed by the BASECOL team, whose members are the scientific co-authors of this publication. Whenever there is only one available data set, this set is

\footnotetext{
http://www . astro.uni - koeln.de/cdms

6 http://spec.jpl.nasa.gov/

7 http://www. cfa.harvard.edu/hitran/
} 
recommended except if there are some obvious problems with the calculations. Whenever there are more data sets available, the recommendation is based on the level of theory used in the calculations.

In addition to the level of theory, the quality of the fitting procedure for the PES and the quality of the dynamical calculations is assessed as far as the information provided by the authors allows. For example in CC/CS dynamical calculations, different sources of inaccuracies can emerge: when cross sections are provided on an inadequate grid of collision energies, then the corresponding rate coefficients may be inaccurate, particularly at low and high temperatures; there might be lack of convergence of the basis sets used in the calculation, lack of convergence of the cross sections with respect to total angular momentum, or lack of convergence in the propagation parameters of the differential equation. All these sources of inaccuracies should be carefully checked for different energies and transitions, and in principle an estimate of inaccuracies should be provided by the authors. In addition the authors should provide an assessment of the quality of the potential energy surfaces (see Sect. 2.2.2), as well as comparisons with experimental data when available. The flag "Recommended", indicated on BASECOL interface, is assessed on the basis of the quality information provided by the authors, and the explanation about recommendation is given in the information section associated with each collisional set.

\section{Technical characteristics and interoperability through VAMDC}

The BASECOL2012 database is freely accessible from http:// basecol.vamdc. $\mathrm{eu}^{8}$ and is currently maintained and mirrored in two geographically distinct locations of Paris Observatory. This ensures long-term maintenance of services and 24-h availability.

Each data set is labeled by a version number with the date of "publication" or "modification" of the data set on the BASECOL2012 website. A modification would correspond to errors found in the datasets after the date of BASECOL publication. Modification of the data description does not lead to a new version of the data set. The history of version is given at the bottom of each data set main webpage. The version number, together with the corresponding date of publication or modification, is indicated in the data set's file name, which can be downloaded in ASCII. The version of external spectroscopic data follows the version procedure of the original database.

BASECOL2012 is interoperable within the Virtual Atomic and Molecular Data Center (VAMDC) (Dubernet et al. 2010; Endres et al. 2011; Rixon et al. 2011); i.e., it is interoperable with the current 24 atomic and molecular databases registered within VAMDC and can be queried by any client that implements the VAMDC standards ${ }^{9}$.

In particular, BASECOL2012 is accessible from the VAMDC portal $^{10}$ and from the SPECTCOL tool (Dubernet et al. 2012; Dubernet \& Nenadovic 2011) ${ }^{11}$; that allow a user to mix any sets of BASECOL collisional rate coefficients with any sets of spectroscopic data. The SPECTCOL tool evolves with user requirements, and the last version includes the possibilities of

\footnotetext{
8 Equivalent to http://basecol.vamdc.org, http://basecol. obspm.fr, http://basecol.obs-besancon.fr

9 http://www. vamdc.eu/standards

10 www. vamdc.eu/portal

${ }^{11}$ www.vamdc.eu/software
}

scaling the collisional rate coefficients, output of the data in the RADEX format (van der Tak et al. 2007) and of discarding quantum numbers when matching BASECOL energy levels with external databases energy levels. This last feature is very useful because the XSAMS schema ${ }^{12}$ allows redundant information to be transferred.

\section{Perspectives}

A well-maintained database is an evolving product, and some new features are foreseen. The "Publication" section will be upgraded with regular notes concerning new data and/or evaluation. Once the VAMDC standards are more stable, the BASECOL energy levels (section "Labelling Energy levels"), rate coefficients (section "Rate Coefficients"), and the combined "Radiation/Collision" data will be emitted in XSAMS format for download. We intend to provide a password-protected administrative interface enabling scientists to upload their most recent results themselves. As a service to the astrophysics community, we also intend to create some in-house combined collisional sets that will include extrapolations in order to cover the full range of temperatures and transitions necessary for the astrophysical applications.

Acknowledgements. BASECOL is a collaborative project supported by the CNRS with Programme National PCMI (Physique Chimie du Milieu Interstellaire) and VO France, The scientific council of the Paris Observatory, ASTRONET/INSU, LERMA. We acknowledge the help of people that previously contributed to the development of BASECOL, and especially Gautier Souesme and Bernard Debray. We also thank all the scientists who have made their results available to us. For the implementation of BASECOL within VAMDC, we acknowledge VAMDC. VAMDC is funded under the "Combination of Collaborative Projects and Coordination and Support Actions" Funding Scheme of EU-FP7 Program, call topic INFRA-2008-1.2.2 Scientific Data Infrastructure and Grant Agreement number 239108.

\section{Appendix A: Comments on some collisional systems}

\section{A.1. Excitation of $\mathrm{H}_{2}$}

In some $\mathrm{CC}$ or $\mathrm{CS}$ calculations, some collisional sets have been obtained with rotational or rovibrational basis sets that are missing a few energy levels, because the basis set cuts were performed using the principal rotational quantum number instead of using a cut at a given energy. This is the case for $\mathrm{H}_{2}$ calculations and BASECOL has replaced the corresponding missing collisional transitions by a value of zero for the rate coefficients for the whole range of temperature. For collision identifier (ID) 24 and 26 (see Col. 1 of Table A.1), the rate coefficients have not been calculated for transitions involving level 24 of ortho$\mathrm{H}_{2}$, (i.e. $v=1, j=11$ ), but are available for transitions involving level $25(v=3, j=7)$. Therefore all rate coefficients involving level 24 are set to zero. For ID $=25,27,31$, the rate coefficients have not been calculated for transitions involving level 27 of para- $\mathrm{H}_{2}$, (i.e. $v=1, j=14$ ), but are available for transitions involving level $28(v=3, j=8)$. Therefore all rate coefficients involving level 27 are set to zero. For ID $=28$ the rate coefficients have not been calculated for transitions involving levels 18,20 of ortho- $\mathrm{H}_{2}$, (i.e. $v=3, j=1 ; v=3, j=3$ ). Therefore all rate coefficients involving levels 18, 20 are set to zero.

12 http://vamdc.eu/standards 


\section{A.2. Excitation of $\mathrm{CH}_{3} \mathrm{OH}$}

Quantum numbers and symmetries used to label the energy levels of methanol have not been agreed on yet within VAMDC and therefore the notation of Rabli \& Flower (2010a,b, 2011) are used in BASECOL. This notation might evolve in the future.

\section{A.3. Excitation of $\mathrm{H}_{3} \mathrm{O}^{+}$by electrons}

The collision sets of o/p- $\mathrm{H}_{3} \mathrm{O}^{+}$(Faure \& Tennyson 2003) have incomplete transitions because the authors considered only the $K=0$ and $K=1$ ladders. The authors expect to complete the original calculations by adding the $K>1$ levels and extending the temperatures down to $10 \mathrm{~K}$.

\section{A.4. Excitation of $\mathrm{H}_{3}^{+}$by electrons}

The recommended sets of $\mathrm{o} / \mathrm{p}-\mathrm{H}_{3}^{+}$by electrons (Kokoouline et al. 2010) use a complete set of energy levels up to the 7 th rotational level for $\mathrm{o}-\mathrm{H}_{3}{ }^{+}$and up to the 13 th rotational level for $\mathrm{p}-\mathrm{H}_{3}^{+}$. Nevertheless, the collisional sets miss some transitions between energy levels. The missing collisional rate coefficients can be set equal to zero as the missing rate coefficients are typically three orders of magnitude smaller than the given collisional data.

\section{A.5. Excitation of o- $\mathrm{H}_{2} \mathrm{O} / \mathrm{p}-\mathrm{H}_{2} \mathrm{O}$ by electrons}

The collision rotational sets of o/p- $\mathrm{H}_{2} \mathrm{O}$-electron (Faure et al. 2004a) are only complete among the first 14th rotational levels. The user is advised to use the ro-vibrational collisional sets of Faure \& Josselin (2008) for transitions from levels above the 14 th rotational level and for temperature above $200 \mathrm{~K}$.

\section{A.6. Excitation of $\mathrm{SiC}_{2}$ and $\mathrm{c}-\mathrm{C}_{3} \mathrm{H}_{2}$}

The work of Green et al. (1987) on excitation of $\mathrm{c}-\mathrm{C}_{3} \mathrm{H}_{2}$ by $\mathrm{He}$ has been used by Chandra \& Kegel (2000) to provide state-tostate rate coefficients between the 47 lowest ortho levels and the 48 lowest para levels of $\mathrm{c}-\mathrm{C}_{3} \mathrm{H}_{2}$ colliding with $\mathrm{H}_{2}$, for $T=30$, $60,90,120 \mathrm{~K}$. In the same paper, the work of Palma \& Green (1987) on excitation of $\mathrm{SiC}_{2}$ by $\mathrm{He}$ has been used to provide state-to-state rate coefficients among the lowest 40 energy levels of $\mathrm{SiC}_{2}$ colliding with $\mathrm{H}_{2}$ and for temperature $T=25,50$, $75,100,125 \mathrm{~K}$. In BASECOL the calculations have been redone using the same ingredients as Chandra \& Kegel (2000), and we found that Chandra \& Kegel (2000) do not provide rate coefficients for collisions of $\mathrm{c}-\mathrm{C}_{3} \mathrm{H}_{2}$ and $\mathrm{SiC}_{2}$ with $\mathrm{H}_{2}$, as the title of their paper indicates, but instead collisions with He. The LAMDA database Schöier et al. (2005) gives the wrong indication on this matter.

\section{A.7. Excitation of o- $\mathrm{H}_{2} \mathrm{O} / \mathrm{p}-\mathrm{H}_{2} \mathrm{O}$ by $\mathrm{He}, \mathrm{H}_{2}$ and recommendation}

Owing to its importance in astrophysical studies, the calculation of collisional rate coefficients for the water molecule have been refined through the past decades, and many sets are available for this molecule. The first set that was available to the astrophysicists considered $\mathrm{He}$ as a collisional partner (Green et al. 1993). This set can still be considered as a reference, but only if collisions with $\mathrm{He}$ are considered explicitly in the modelling. This set of rates must not be used to emulate collisions with $\mathrm{H}_{2}$ by using an ad hoc scaling factor. The specific consideration of $\mathrm{H}_{2}$ as a collisional partner was first considered by Phillips et al. (1996). However, these early calculations, which covered a reduced number of temperatures and water energy levels, have been outclassed due to the calculation of a most accurate PES (Faure et al. 2005; Valiron et al. 2008), which has been used to calculate collisional rate coefficients with the QCT method (Faure et al. 2007b) and with the quantum CC approach (Dubernet et al. 2009; Daniel et al. 2010, 2011). This latter set is the most accurate and outclass the QCT set. We note that the highest temperature considered in the quantum set is $1500 \mathrm{~K}$ while the QCT calculation extend to $2000 \mathrm{~K}$. These highest temperatures can be of interest in the study of the envelopes of AGB stars (Royer et al. 2010; Neufeld et al. 2011) or to study shock regions (Nisini et al. 2010; Flower \& Pineau des Forêts 2012). For the range of temperature 1500-2000 K, we still recommend using the quantum set rather than the QCT set, by extrapolating the collisional rate coefficients. Indeed, the comparison of the QCT and quantum rate coefficients made in Daniel et al. (2012) showed that even at high temperature, the QCT rate coefficients and quantum thermalized rate coefficients can predict intensities that differ by up to a factor 3 , an error which can be directly linked to the difference in the magnitude of the rate coefficients which is also typically of a factor 3 . Extrapolating the quantum rate coefficients should thus be more reliable.

Additionally, Faure \& Josselin (2008) provide ro-vibrational rate coefficients for the first five vibrational levels of both $0-\mathrm{H}_{2} \mathrm{O}$ and $\mathrm{p}-\mathrm{H}_{2} \mathrm{O}$ and for the range of temperature $200-5000 \mathrm{~K}$. Due to the high temperatures considered by this set, it is still recommended when the ro-vibrational excitation has to be considered. However, for the range of temperature covered by the quantum CC calculations in $(v 1, v 2, v 3)=(0,0,0)$, i.e. $T k=20-1500 \mathrm{~K}$, this set must not be used and the quantum CC calculations should be used instead. Indeed, for the rotational transitions among a given vibrational state, the rate coefficients of the Faure \& Josselin (2008) set are taken to be equal to the QCT rates of Faure et al. (2007b) in $(v 1, v 2, v 3)=(0,0,0)$. These rates can be directly replaced by the quantum $\mathrm{CC}$ rate coefficients. For the collisional transitions between different vibrational states, the Faure \& Josselin (2008) rates still correspond to the QCT rate coefficients of Faure et al. (2007b) but are scaled by a factor $P\left(v-v^{\prime}\right)$ which has been determined experimentally. In this case, we recommend to use the quantum $C C$ rate coefficients and multiply these rates by the factor $P\left(v-v^{\prime}\right)$ provided by Faure \& Josselin (2008). 


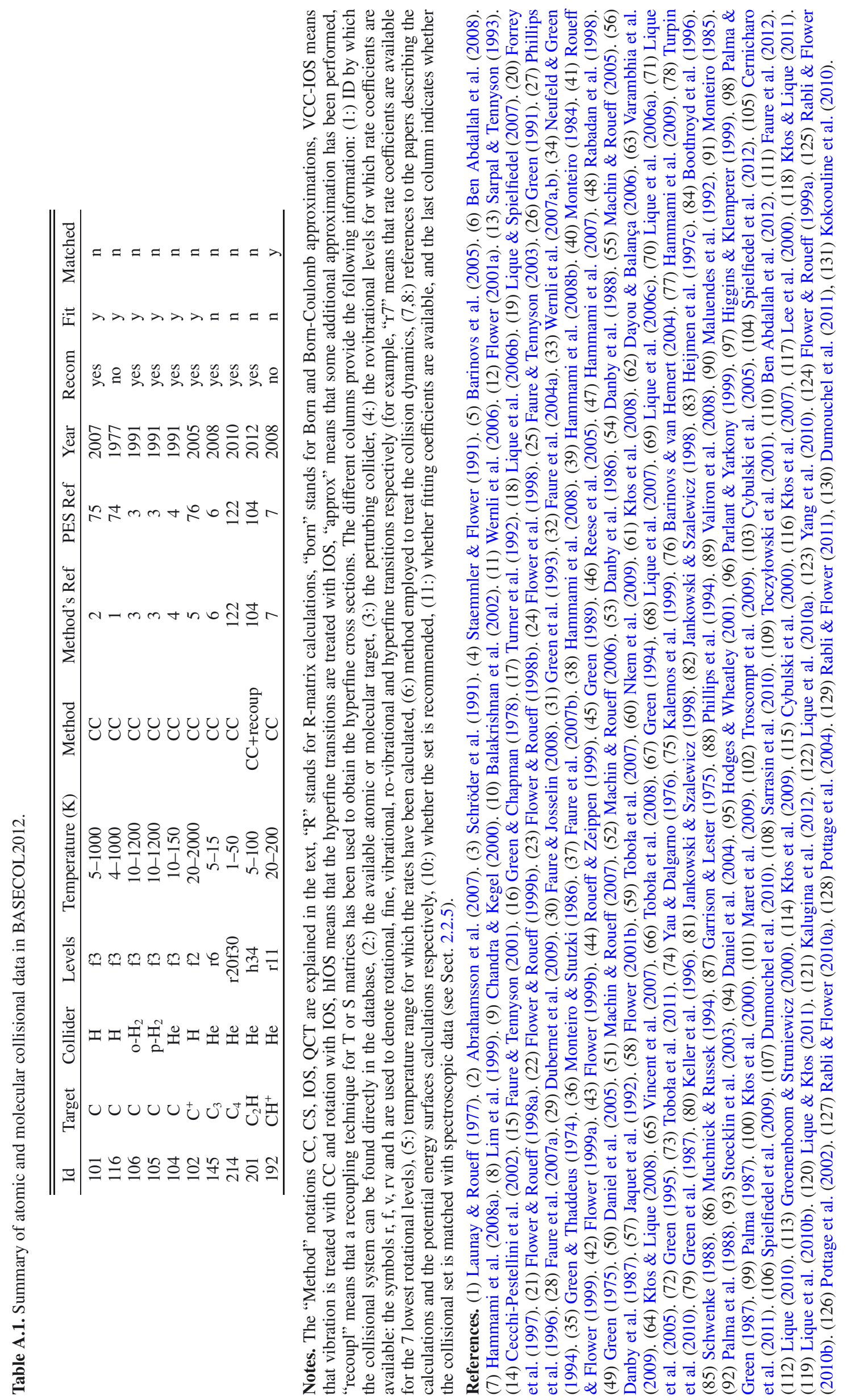




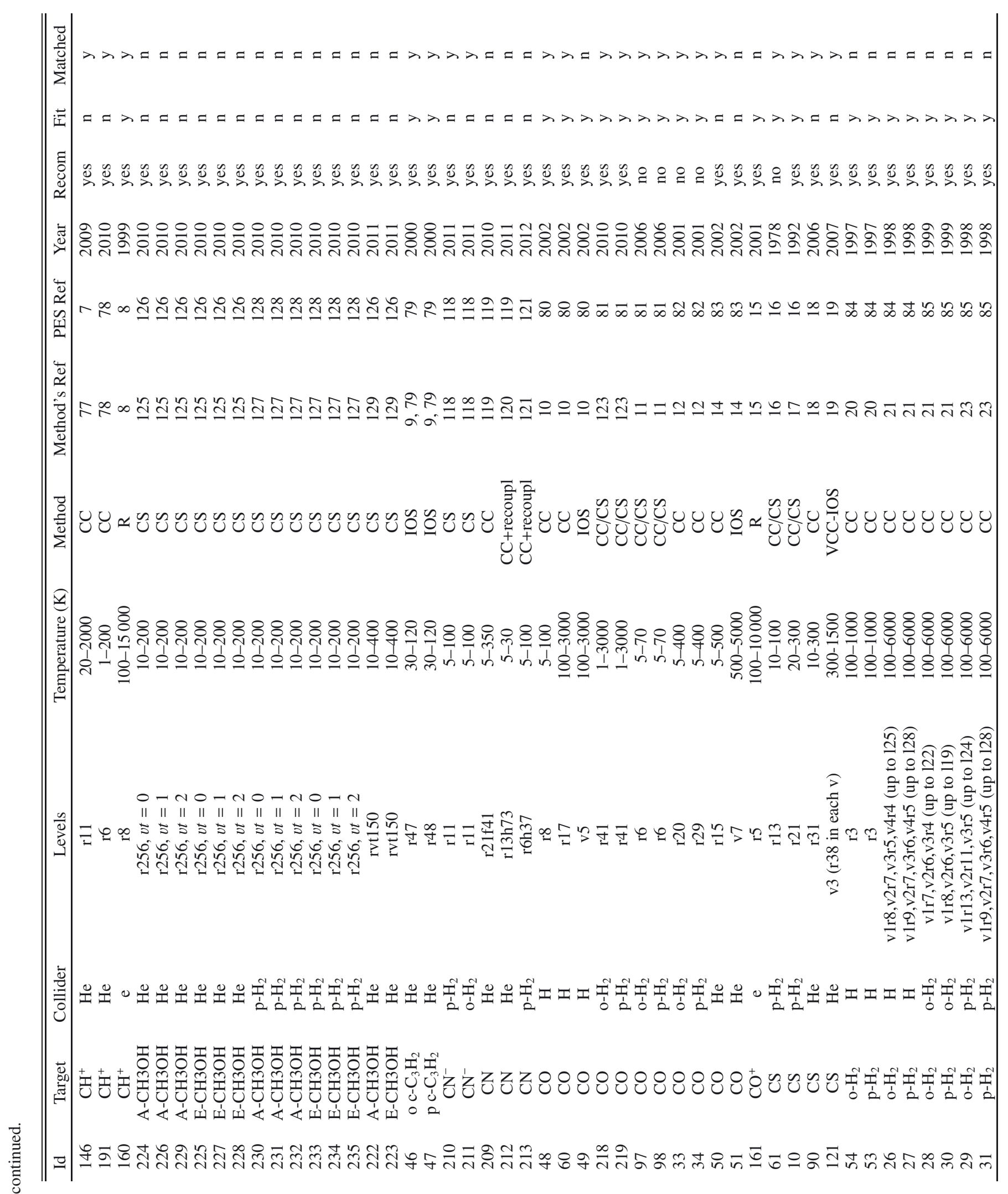




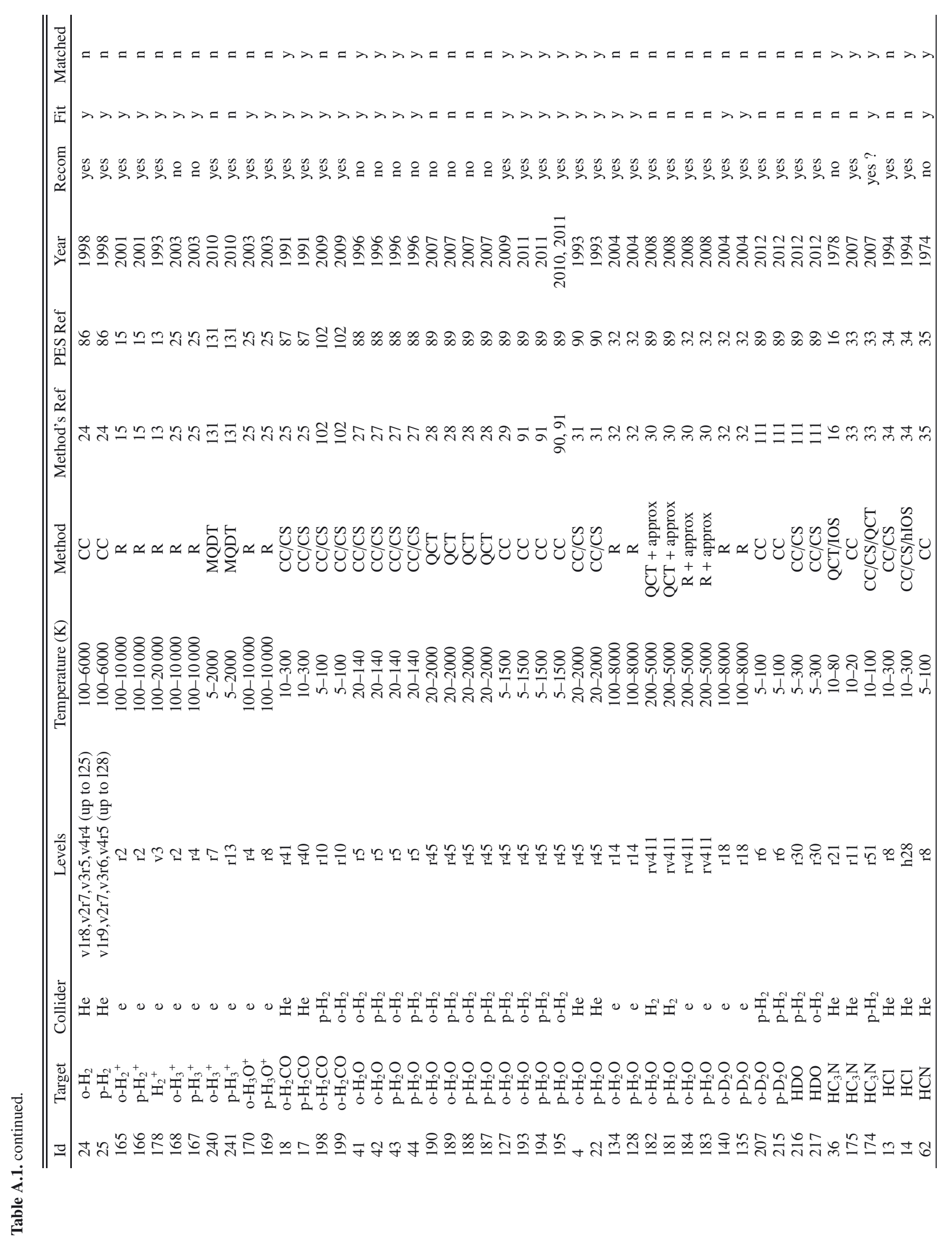




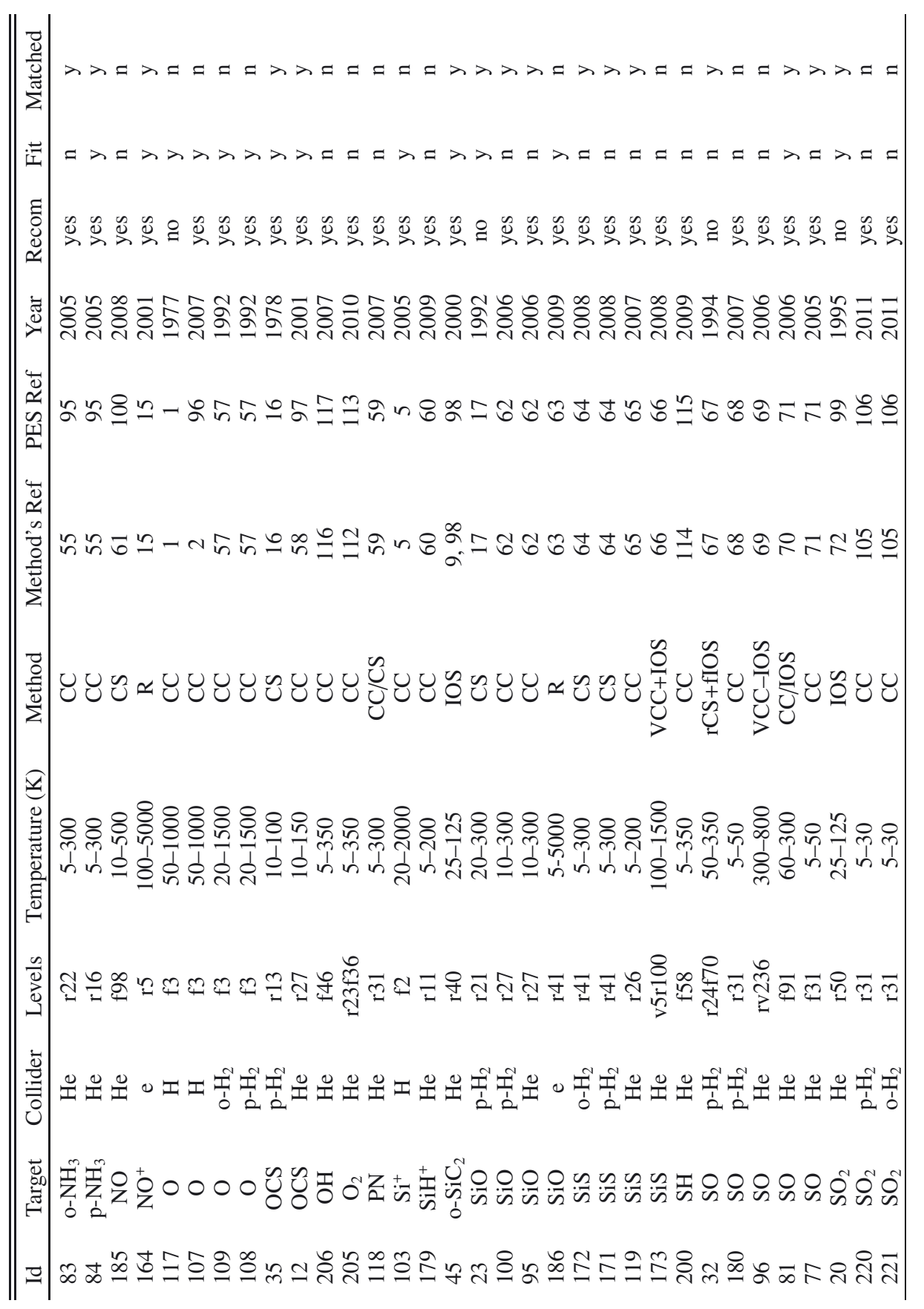




\section{References}

Abrahamsson, E., Krems, R. V., \& Dalgarno, A. 2007, ApJ, 654, 1171 Alexander, M. 1985, Chem. Phys., 92, 337

Arthurs, A. M., \& Dalgarno, A. 1960, Proc. R. Soc. A, 256, 540 Baer, M. 1975, Chem. Phys. Lett., 35, 112

Balakrishnan, N., Yan, M., \& Dalgarno, A. 2002, ApJ, 568, 443

Barinovs, G., \& van Hemert, M. 2004, Chem. Phys. Lett., 399, 406

Barinovs, Ğ., van Hemert, M. C., Krems, R., \& Dalgarno, A. 2005, ApJ, 620, 537

Bemish, R., Oudejans, L., Miller, R., et al. 1998, J. Chem. Phys., 109, 8968 Ben Abdallah, D., Hammami, K., Najar, F., et al. 2008, ApJ, 686, 379

Ben Abdallah, D., Najar, F., Jaidane, N., Dumouchel, F., \& Lique, F. 2012, MNRAS, 419, 2441

Bergin, E. A., Phillips, T. G., Comito, C., et al. 2010, A\&A, 521, L20

Boothroyd, A. I., Keogh, W. J., Martin, P. G., \& Peterson, M. R. 1996, J. Chem. Phys., 104, 7139

Braams, B. J., \& Bowman, J. M. 2009, Int. Rev. Phys. Chem., 28, 577

Bukowski, R., Szalewicz, K., Groenenboom, G., \& Van der Avoird, A. 2007, Science, 315, 1249

Bukowski, R., Cencek, W., Jankowski, P., et al. 2008, SAPT2008: An Ab Initio Program for Many-Body Symmetry-Adapted Perturbation Theory Calculations of Intermolecular Interaction Energies (University of Delaware and University of Warsaw)

Caux, E., Kahane, C., Castets, A., et al. 2011, A\&A, 532, A23

Ceccarelli, C., Bacmann, A., Boogert, A., et al. 2010, A\&A, 521, L22

Cecchi-Pestellini, C., Bodo, E., Balakrishnan, N., \& Dalgarno, A. 2002, ApJ, 571,1015

Cernicharo, J., Spielfiedel, A., Balança, C., et al. 2011, A\&A, 531, A103

Chandra, S., \& Kegel, W. H. 2000, A\&AS, 142, 113

Cybulski, S. M., Toczylowski, R. R., Lee, H.-S., \& McCoy, A. B. 2000, J. Chem. Phys., 113, 9549

Cybulski, S. M., Krems, R. V., Sadeghpour, H. R., et al. 2005, J. Chem. Phys., 122,094307

Dagdigian, P., \& Alexander, M. H. 2011, J. Chem. Phys., 135, 064306

Danby, G., Flower, D. R., Kochanski, E., Kurdi, L., \& Valiron, P. 1986, J. Phys. B At. Mol. Opt. Phys., 19, 2891

Danby, G., Flower, D. R., Valiron, P., Kochanski, E., \& Kurdi, L. 1987, J. Phys. B At. Mol. Opt. Phys., 20, 1039

Danby, G., Flower, D. R., Valiron, P., Schilke, P., \& Walmsley, C. M. 1988, MNRAS, 235, 229

Daniel, F., Dubernet, M.-L., \& Meuwly, M. 2004, J. Chem. Phys., 121, 4540

Daniel, F., Dubernet, M.-L., Meuwly, M., Cernicharo, J., \& Pagani, L. 2005, MNRAS, 363, 1083

Daniel, F., Dubernet, M.-L., Pacaud, F., \& Grosjean, A. 2010, A\&A, 517, A13

Daniel, F., Dubernet, M.-L., \& Grosjean, A. 2011, A\&A, 536, A76

Daniel, F., Goicoechea, J. R., Cernicharo, J., Dubernet, M.-L., \& Faure, A. 2012, A\&A, 547, A81

Dayou, F., \& Balança, C. 2006, A\&A, 459, 297

de Graauw, T., Helmich, F. P., Phillips, T. G., et al. 2010, A\&A, 518, L6

DePristo, A. E., \& Alexander, M. H. 1976, J. Chem. Phys., 64, 3009

Drouin, B., \& Wiesenfeld, L. 2012, Phys. Rev. A, 86, 2705

Drouin, B. J., Pearson, J. C., \& Dick, M. J. 2010, Phys. Rev. A, 82, 6704

Dubernet, M.-L., \& Nenadovic, L. 2011, in Astrophysics Source Code Library, ascl:1111.005

Dubernet, M. L., Grosjean, A., Flower, D., et al. 2006, J. Plasma Res. Ser., 7, 356

Dubernet, M.-L., Daniel, F., Grosjean, A., \& Lin, C. Y. 2009, A\&A, 497, 911

Dubernet, M. L., Boudon, V., Culhane, J. L., et al. 2010, J. Quant. Spec. Radiat. Transf., 111, 2151

Dubernet, M. L., Roueff, E., Stoecklin, T., Daniel, F., \& Grosjean, A. 2011, in EAS Publ. Ser. 52, eds. M. Röllig, R. Simon, V. Ossenkopf, \& J. Stutzki, 217 Dubernet, M. L., Nenadovic, L., \& Doronin, M. 2012, in ASP Conf. Ser., TBD, ADASS XXI, eds. P. Ballester, \& D. Egret (San Francisco: ASP), TBD

Dumouchel, F., Faure, A., \& Lique, F. 2010, MNRAS, 406, 2488

Dumouchel, F., Kłos, J., \& Lique, F. 2011, PCCP, 13, 8204

Endres, C., Giesen, T., Muller, H., et al. 2011, in IAU Symp., 280, 333

Faure, A., \& Josselin, E. 2008, A\&A, 492, 257

Faure, A., \& Tennyson, J. 2001, MNRAS, 325, 443

Faure, A., \& Tennyson, J. 2003, MNRAS, 340, 468

Faure, A., Gorfinkiel, J. D., \& Tennyson, J. 2004a, MNRAS, 347, 323

Faure, A., Gorfinkiel, J. D., \& Tennyson, J. 2004b, J. Phys. B: At. Mol. Opt. Phys., 37, 801

Faure, A., Valiron, P., Wernli, M., et al. 2005, J. Chem. Phys., 122, 221102

Faure, A., Kokoouline, V., Greene, C. H., \& Tennyson, J. 2006, J. Phys. B: At. Mol. Opt. Phys., 39, 4261

Faure, A., Crimier, N., Ceccarelli, C., et al. 2007a, A\&A, 472, 1029

Faure, A., Varambhia, H. N., Stoecklin, T., \& Tennyson, J. 2007b, MNRAS, 382, 840
Faure, A., Wiesenfeld, L., Scribano, Y., \& Ceccarelli, C. 2012, MNRAS, 420, 699

Flower, D. 2007, Molecular Collisions in the Interstellar Medium, 2nd edn. (Cambridge Astrophysics Series), 196

Flower, D. R. 1999a, J. Phys. B At. Mol. Opt. Phys., 32, 1755

Flower, D. R. 1999b, MNRAS, 305, 651

Flower, D. R. 2001a, J. Phys. B At. Mol. Opt. Phys., 34, 2731

Flower, D. R. 2001b, MNRAS, 328, 147

Flower, D. R., \& Pineau des Forêts, G. 2012, MNRAS, 421, 2786

Flower, D. R., \& Roueff, E. 1998a, J. Phys. B At. Mol. Opt. Phys., 31, L955

Flower, D. R., \& Roueff, E. 1998b, J. Phys. B At. Mol. Opt. Phys., 31, 2935

Flower, D. R., \& Roueff, E. 1999a, MNRAS, 309, 833

Flower, D. R., \& Roueff, E. 1999b, J. Phys. B At. Mol. Opt. Phys., 32, 3399

Flower, D. R., Roueff, E., \& Zeippen, C. J. 1998, J. Phys. B At. Mol. Opt. Phys., 31,1105

Flower, D. R., Gourhis, G., \& Launay, J.-M. 2000, Comp. Phys. Comm., 131, 187

Forrey, R. C., Balakrishnan, N., Dalgarno, A., \& Lepp, S. 1997, ApJ, 489, 1000

Garrison, B. J., \& Lester, W. A. 1975, J. Chem. Phys., 63, 1449

Gianturco, F. A., \& Jain, A. 1986, Phys. Rep., 143, 347

Ginard, D., González-García, M., Fuente, A., et al. 2012, A\&A, 543, A27

Green, S. 1975, ApJ, 201, 366

Green, S. 1989, ApJS, 70, 813

Green, S. 1991, ApJS, 76, 979

Green, S. 1994, ApJ, 434, 188

Green, S. 1995, ApJS, 100, 213

Green, S., \& Chapman, S. 1978, ApJS, 37, 169

Green, S., \& Thaddeus, P. 1974, ApJ, 191, 653

Green, S., Defrees, D. J., \& McLean, A. D. 1987, ApJS, 65, 175

Green, S., Maluendes, S., \& McLean, A. D. 1993, ApJS, 85, 181

Groenenboom, G. C., \& Struniewicz, I. M. 2000, J. Chem. Phys., 113, 9562

Hammami, K., Lique, F., Jaïdane, N., et al. 2007, A\&A, 462, 789

Hammami, K., Nkem, C., Owono Owono, L. C., Jaidane, N., \& Ben Lakhdar, Z. 2008, J. Chem. Phys., 129, 204305

Hammami, K., Owono, L. O., Jaidane, N., \& Lakhdar, Z. B. 2008a, J. Mol. Struct.: THEOCHEM, 853, 18

Hammami, K., Owono, L. O., Jaidane, N., \& Lakhdar, Z. B. 2008b, J. Mol. Struct.: THEOCHEM, 860, 45

Hammami, K., Owono Owono, L. C., \& Stäuber, P. 2009, A\&A, 507, 1083

Heijmen, T., Moszynski, R., Wormer, P., \& Van der Avoird, A. 1996, Mol. Phys., 89,81

Heijmen, T., Korona, T., Moszynski, R., Wormer, P., \& Van der Avoird, A. 1997a, J. Chem. Phys., 107, 902

Heijmen, T., Moszynski, R., Wormer, P., \& Van der Avoird, A. 1997b, J. Chem. Phys., 107, 9921

Heijmen, T. G. A., Moszynski, R., Wormer, P. E. S., \& van der Avoird, A. 1997c, J. Chem. Phys., 107, 9921

Heijmen, T., Moszynski, R., Wormer, P., et al. 1999a, J. Chem. Phys., 111, 2519

Heijmen, T., Wormer, P., Van der Avoird, A., Miller, R., \& Moszynski, R. 1999b, J. Chem. Phys., 110, 5639

Higgins, K., \& Klemperer, W. 1999, J. Chem. Phys., 110, 1383

Hodges, M. P., \& Wheatley, R. J. 2001, J. Chem. Phys., 114, 8836

Hutson, J. M., \& Green, S. 1994, MOLSCAT computer code,version 14 (UK: Collaborative Computational Project No. 6 of the Science and Engineering Research Council)

Itikawa, Y. 2004, J. Phys. B: At. Mol. Opt. Phys., 37, R1

Itikawa, Y., \& Mason, N. 2005, Phys. Rep., 414, 1

Jankowski, P., \& Szalewicz, K. 1998, J. Chem. Phys., 108, 3554

Jankowski, P., McKellar, A., \& Szalewicz, K. 2012, science, 336, 1147

Jaquet, R., Staemmler, V., Smith, M. D., \& Flower, D. R. 1992, J. Phys. B At. Mol. Opt. Phys., 25, 285

Jeziorski, B., Moszyński, R., \& Szalewicz, K. 1994, Chem. Rev., 94, 1887

Kalemos, A., Mavridis, A., \& Metropoulos, A. 1999, J. Chem. Phys., 111, 9536

Kalugina, Y., Lique, F., \& Kłos, J. 2012, MNRAS, 2545

Keller, H.-M., Floethmann, H., Dobbyn, A. J., et al. 1996, J. Chem. Phys., 105, 4983

Kłos, J., \& Lique, F. 2008, MNRAS, 390, 239

Kłos, J., \& Lique, F. 2011, MNRAS, 418, 271

Kłos, J., Chalasinski, G., Berry, M. T., Bukowski, R., \& Cybulski, S. M. 2000, J. Chem. Phys., 112, 2195

Kłos, J., Lique, F., \& Alexander, M. H. 2007, Chem. Phys. Lett., 445, 12

Kłos, J., Lique, F., \& Alexander, M. H. 2008, Chem. Phys. Lett., 455, 1

Kłos, J., Lique, F., \& Alexander, M. H. 2009, Chem. Phys. Lett., 476, 135

Kokoouline, V., Faure, A., Tennyson, J., \& Greene, C. H. 2010, MNRAS, 405, 1195

Kouri, D. J. 1979, in Atom-molecule collision theory: a guide for the experimentalist, ed. R. B. Bernstein (New York: Plenum Press), 259

Krupnov, A. F. 2010, Phys. Rev. A, 82, 6703 
Launay, J. M. \& Roueff, E. 1977, A\&A, 56, 289

Lee, H., McCoy, A. B., Toczyłowski, R. R., \& Cybulski, S. M. 2000, J. Chem. Phys., 113, 5736

Light, J. C. 1979, in Atom-molecule collision theory: a guide for the experimentalist, ed. R. B. Bernstein (New York: Plenum Press), 239

Lim, A. J., Rabadán, I., \& Tennyson, J. 1999, MNRAS, 306, 473

Lique, F. 2010, J. Chem. Phys., 132, 4311

Lique, F., \& Kłos, J. 2011, MNRAS, 413, L20

Lique, F., \& Spielfiedel, A. 2007, A\&A, 462, 1179

Lique, F., Spielfiedel, A., Dubernet, M.-L., \& Feautrier, N. 2005, J. Chem. Phys., 123,134316

Lique, F., Dubernet, M.-L., Spielfiedel, A., \& Feautrier, N. 2006a, A\&A, 450, 399

Lique, F., Spielfiedel, A., \& Cernicharo, J. 2006b, A\&A, 451, 1125

Lique, F., Spielfiedel, A., Dhont, G., \& Feautrier, N. 2006c, A\&A, 458, 331

Lique, F., Senent, M.-L., Spielfiedel, A., \& Feautrier, N. 2007, J. Chem. Phys., 126,164312

Lique, F., Kłos, J., \& Hochlaf, M. 2010a, PCCP, 12, 15672

Lique, F., Spielfiedel, A., Feautrier, N., et al. 2010b, J. Chem. Phys., 132, 4303

Machin, L., \& Roueff, E. 2005, J. Phys. B At. Mol. Opt. Phys., 38, 1519

Machin, L., \& Roueff, E. 2006, A\&A, 460, 953

Machin, L., \& Roueff, E. 2007, A\&A, 465, 647

Maluendes, S., McLean, A. D., \& Green, S. 1992, J. Chem. Phys., 96, 8150

Maret, S., Faure, A., Scifoni, E., \& Wiesenfeld, L. 2009, MNRAS, 399, 425

McBane, G. 2004, MOLSCAT computer code, parallel version (USA: G. McBane)

McGuire, P., \& Kouri, D. J. 1974, J. Chem. Phys., 60, 2488

Miller, R., Heijmen, T., Wormer, P., Van der Avoird, A., \& Moszynski, R. 1999, J. Chem. Phys., 110, 5651

Monteiro, T. 1984, MNRAS, 210, 1

Monteiro, T. S. 1985, MNRAS, 214, 419

Monteiro, T. S., \& Stutzki, J. 1986, MNRAS, 221, 33P

Moreau, N., \& Dubernet, M. L. 2006a, in SF2A-2006: Semaine de l'Astrophysique Francaise, eds. D. Barret, F. Casoli, G. Lagache, A. Lecavelier, \& L. Pagani, 95

Moreau, N., \& Dubernet, M. L. 2006b, in Astronomical Data Analysis Software and Systems XV, eds. C. Gabriel, C. Arviset, D. Ponz, \& S. Enrique, ASP Conf. Ser., 351, 391

Moszynski, R., Korona, T., Wormer, P., \& Van der Avoird, A. 1997, jpca, 101, 4690

Muchnick, P., \& Russek, A. 1994, J. Chem. Phys., 100, 4336

Müller, H. S. P., Schlöder, F., Stutzki, J., \& Winnewisser, G. 2005, J. Mol. Struct., 742,215

Müller, H. S. P., Thorwirth, S., Roth, D. A., \& Winnewisser, G. 2001, A\&A, 370, L49

Neufeld, D. A., \& Green, S. 1994, ApJ, 432, 158

Neufeld, D. A., González-Alfonso, E., Melnick, G., et al. 2011, ApJ, 727, L29

Nisini, B., Benedettini, M., Codella, C., et al. 2010, A\&A, 518, L120

Nkem, C., Hammami, K., Manga, A., et al. 2009, J. Mol. Struct.: Theochem., 901, 220

Pack, R. T. 1974, J. Chem. Phys., 60, 633

Palma, A. 1987, ApJS, 64, 565

Palma, A., \& Green, S. 1987, ApJ, 316, 830

Palma, A., Green, S., Defrees, D. J., \& McLean, A. D. 1988, J. Chem. Phys., 89, 1401

Parlant, G., \& Yarkony, D. R. 1999, J. Chem. Phys., 110, 363

Patel, N. A., Young, K. H., Gottlieb, C. A., et al. 2011, ApJS, 193, 17

Pattengill, M. D. 1979, in Atom-molecule collision theory: a guide for the experimentalist, ed. R. B. Bernstein (New York: Plenum Press), 265

Phillips, T. R., Maluendes, S., \& Green, S. 1996, ApJS, 107, 467

Phillips, T. R., Maluendes, S., McLean, A. D., \& Green, S. 1994, J. Chem. Phys., 101,5824

Pickett, H. M., Poynter, R. L., Cohen, E. A., et al. 1998, J. Quant. Spectrosc., \& Rad. Transfer, 60, 883
Pottage, J. T., Flower, D. R., \& Davis, S. L. 2002, J. Phys. B: At. Mol. Opt. Phys., 35,2541

Pottage, J. T., Flower, D. R., \& Davis, S. L. 2004, MNRAS, 352, 39

Rabadan, I., Sarpal, B. K., \& Tennyson, J. 1998, MNRAS, 299, 171

Rabitz, H. 1976, in Dynamics of Molecular Collisions, Part A, ed. W. H. Miller (New York: Plenum Press), 33

Rabli, D., \& Flower, D. R. 2010a, MNRAS, 406, 95

Rabli, D., \& Flower, D. R. 2010b, MNRAS, 403, 2033

Rabli, D., \& Flower, D. R. 2011, MNRAS, 411, 2011

Reese, C., Stoecklin, T., Voronin, A., \& Rayez, J. C. 2005, A\&A, 430, 1139

Rixon, G., Dubernet, M. L., Piskunov, N., et al. 2011, in AIP Conf. Ser., 1344, eds. A. Bernotas, R. Karazija, \& Z. Rudzikas, 107

Rothman, L. S., Gordon, I. E., Barbe, A., et al. 2009, JQSRT, 110, 533

Roueff, E., \& Flower, D. R. 1999, MNRAS, 305, 353

Roueff, E., \& Zeippen, C. J. 1999, A\&A, 343, 1005

Royer, P., Decin, L., Wesson, R., et al. 2010, A\&A, 518, L145

Sarpal, B. K., \& Tennyson, J. 1993, MNRAS, 263, 909

Sarrasin, E., Abdallah, D. B., Wernli, M., et al. 2010, MNRAS, 404, 518

Schöier, F. L., van der Tak, F. F. S., van Dishoeck, E. F., \& Black, J. H. 2005, A\&A, 432, 369

Schröder, K., Staemmler, V., Smith, M. D., Flower, D. R., \& Jaquet, R. 1991, J. Phys. B At. Mol. Opt. Phys., 24, 2487

Schwenke, D. W. 1988, J. Chem. Phys., 89, 2076

Secrest, D. 1975, J. Chem. Phys., 62, 710

Secrest, D. 1979, in Atom-molecule collision theory: a guide for the experimentalist, ed. R. B. Bernstein (New York: Plenum Press), 265

Shafir, D., Novotny, S., Buhr, H., et al. 2009, Phys. Rev. Lett., 102, 223202

Spielfiedel, A., Senent, M.-L., Dayou, F., et al. 2009, J. Chem. Phys., 131, 014305

Spielfiedel, A., Feautrier, N., Najar, F., et al. 2012, MNRAS, 421, 1891

Staemmler, V., \& Flower, D. R. 1991, J. Phys. B At. Mol. Opt. Phys., 24, 2343

Stoecklin, T., Voronin, A., \& Rayez, J. C. 2003, Chem. Phys., 294, 117

Tennyson, J. 2010, Phys. Rep., 491, 29

Tercero, B., Vincent, L., Cernicharo, J., Viti, S., \& Marcelino, N. 2011, A\&A, 528, A26

Toboła, R., Kłos, J., Lique, F., Chałasiński, G., \& Alexander, M. H. 2007, A\&A, 468,1123

Toboła, R., Lique, F., Kłos, J., \& Chałasiński, G. 2008, J. Phys. B At. Mol. Opt. Phys., 41, 155702

Toboła, R., Dumouchel, F., Kłos, J., \& Lique, F. 2011, J. Chem. Phys., 134, 4305

Toczyłowski, R. R., Doloresco, F., \& Cybulski, S. M. 2001, J. Chem. Phys., 114, 851

Troscompt, N., Faure, A., Wiesenfeld, L., Ceccarelli, C., \& Valiron, P. 2009, A\&A, 493, 687

Tsien, T. P., \& Pack, R. T. 1970a, Chem. Phys. Lett., 6, 54

Tsien, T. P., \& Pack, R. T. 1970b, Chem. Phys. Lett., 6, 400

Turner, B. E., Chan, K.-W., Green, S., \& Lubowich, D. A. 1992, ApJ, 399, 114

Turpin, F., Stoecklin, T., \& Voronin, A. 2010, A\&A, 511, A28

Valiron, P., Wernli, M., Faure, A., et al. 2008, J. Chem. Phys., 129, 134306

van der Tak, F., Black, J., Schöier, F., Jansen, D., \& van Dishoeck, E. 2007, A\&A, 468, 627

Varambhia, H. N., Gupta, M., Faure, A., Baluja, K. L., \& Tennyson, J. 2009, J. Phys. B At. Mol. Opt. Phys., 42, 095204

Vincent, L. F. M., Spielfiedel, A., \& Lique, F. 2007, A\&A, 472, 1037

Werner, H.-J., Knowles, P. J., Knizia, G., Manby, F. R., \& Schütz, M. 2012, WIREs Comput Mol Sci, 2, 242

Wernli, M., Valiron, P., Faure, A., et al. 2006, A\&A, 446, 367

Wernli, M., Wiesenfeld, L., Faure, A., \& Valiron, P. 2007a, A\&A, 464, 1147

Wernli, M., Wiesenfeld, L., Faure, A., \& Valiron, P. 2007b, A\&A, 475, 391

Wiesenfeld, L., \& Faure, A. 2010, Phys. Rev. A, 82, 040702

Yang, B., Stancil, P. C., Balakrishnan, N., \& Forrey, R. C. 2010, ApJ, 718, 1062

Yau, A. W., \& Dalgarno, A. 1976, ApJ, 206, 652

Zernickel, A., Schilke, P., Schmiedeke, A., et al. 2012, A\&A, 546, A87

Zhang, R., Faure, A., \& Tennyson, J. 2009, Phys. Scr., 80, 5301 